\title{
p-ADIC CURVATURE AND A CONJECTURE OF SERRE
}

\author{
BY HOWARD GARLAND
}

Communicated by H. Bass, October 7, 1971

1. In this note we announce a vanishing theorem for the cohomology of discrete subgroups of $p$-adic groups. The methods and results bear a striking analogy with the real case (see Matsushima [4]). In particular, we define " $p$-adic curvature" for the $p$-adic symmetric spaces of Bruhat-Tits (see [1]). As in the real case, we then reduce the proof of our vanishing theorem to the assertion that the minimum eigenvalues of certain $p$-adic curvature transformations are sufficiently large. This last condition can then be verified for "sufficiently large" residue class fields.

Before giving a more detailed description of our results we introduce some notation. Thus let $\boldsymbol{Z}$ denote the ring of rational integers and $\boldsymbol{Q}, \boldsymbol{R}$, and $C$ the fields of rational, real and complex numbers, respectively. For a prime $p, \boldsymbol{Q}_{p}$ will denote the $p$-adic completion of $\boldsymbol{Q}$. More generally $k_{v}$ will denote a nondiscrete, totally disconnected, and locally compact (commutative) field.

Let $\boldsymbol{G}$ denote a simply-connected, linear algebraic group defined and simple over $k_{v}$ and let $\boldsymbol{G}_{\boldsymbol{k}_{v}}$ denote the $k_{\boldsymbol{v}}$-rational points of $\boldsymbol{G}$. Let $V_{\boldsymbol{Q}}$ denote a finite-dimensional vector space over $\boldsymbol{Q}, \Gamma$ an abstract group, and $\rho: \Gamma \rightarrow$ Aut $V_{\boldsymbol{Q}}$ a representation. Let $H^{i}(\Gamma, \rho)$ denote the $i$ th EilenbergMac Lane group of $\Gamma$ with respect to $\rho$. If $V=\boldsymbol{Q}$, and $\rho$ is the trivial representation we write $H^{i}(\Gamma, Q)$ in place of $H^{i}(\Gamma, \rho)$. By a uniform lattice in $\boldsymbol{G}_{\boldsymbol{k}_{v}}$ we mean a discrete subgroup $\Gamma \subset \boldsymbol{G}_{\boldsymbol{k}_{v}}$ such that $\boldsymbol{G}_{\boldsymbol{k}_{v}} / \Gamma$ is compact.

THEOREM 1. For every integer $l$, there is an integer $N(l)$ such that if the residue class field of $k_{v}$, has at least $N(l)$ elements, if $\Gamma \subset \boldsymbol{G}_{k_{v}}$ is a uniform lattice, and if $\rho: \Gamma \rightarrow$ Aut $V_{\mathbf{Q}}$ is a finite-dimensional representation such that $\rho(\Gamma)$ is contained in the orthogonal group of some positive-definite quadratic form on $V_{\mathbf{Q}}$, then $H^{i}(\Gamma, \rho)=0$ for $0<i<l$. In particular, $H^{i}(\Gamma, Q)=0$ for $0<i<l$.

Except for our restriction on the residue class field, this theorem answers a question raised by J.-P. Serre for the trivial representation. It seems likely that sharper estimates for the minimum eigenvalues of $p$-adic curvature will enable one to eliminate the restriction on the residue class field. For nontrivial $\rho$, we have had to introduce the additional restriction that $\rho(\Gamma)$ is contained in the orthogonal group of a positive-definite form on $V_{\boldsymbol{Q}}$. Serre has conjectured that if $\rho$ is obtained from a representation of $\boldsymbol{G}_{\boldsymbol{k}_{v}}$ in

AMS 1970 subject classifications. Primary 20E40, 20G10, 20G25. 
$V_{\mathbf{Q}} \otimes_{\mathbf{Q}} k_{v}$, then this additional restriction is unnecessary. Incidentally, Serre observed that $H^{i}(\Gamma, \rho)=0$ for $i>l$. For $i=l$ we have the following result (also observed by Borel and Serre):

THEOREM 2. If $\Gamma$ is a torsion-free lattice in $\boldsymbol{G}_{\boldsymbol{k}_{v}}$, then $\operatorname{dim}_{\mathbf{Q}} H^{l}(\Gamma, \boldsymbol{Q})$ $=$ multiplicity of the Steinberg representation in $L^{2}\left(\boldsymbol{G}_{k_{v}} / \Gamma\right)$.

(See Shalika [6] for the definition of the Steinberg representation in the Chevalley case - one must make the appropriate translation from the adjoint group to the simply-connected group.) Our main theorem (Theorem 1) together with Theorem 2 yields a partial $p$-adic analogue of the result in Schmid [5].

2. An indication of the proof. Let $\Gamma \subseteq \boldsymbol{G}_{k_{v}}$ be a uniform lattice. For simplicity, assume $\Gamma$ is torsion-free and $\rho$ is the trivial representation (so $V_{\boldsymbol{Q}}=\boldsymbol{Q}$ ). Let $\mathscr{T}$ be the Bruhat-Tits complex associated with $\boldsymbol{G}_{k_{v}}$ (see [1]). Then $\mathscr{T}$ is a simplicial complex and is contractible. $\boldsymbol{G}_{k_{v}}$ acts on $\mathscr{T}$ as a group of simplicial transformations (and we make the convention that $\boldsymbol{G}_{k_{v}}$ acts to the left). The action of $\Gamma$ is then properly discontinuous and fixed-point free. Hence, it follows that

$$
H^{i}(\Gamma, \boldsymbol{Q}) \cong H^{i}(\Gamma \backslash \mathscr{T}, \boldsymbol{Q}) \text {. }
$$

Since $\Gamma \backslash \boldsymbol{G}_{\boldsymbol{k}_{v}}$ is compact, one knows that $\Gamma \backslash \mathscr{T}$ is a finite complex which for simplicity, we assume to be a simplicial.

Following Eckmann and Hodge, we describe a "harmonic theory" for the $\boldsymbol{Q}$-cochains of a finite simplicial complex $\mathscr{S}$. First, for each geometric simplex $\sigma$ of $\mathscr{S}$, fix an oriented simplex $\hat{\sigma}$ corresponding to $\sigma$. By a Riemannian metric on $\mathscr{S}$ we will mean a function from the geometric simplices to the positive real numbers. We fix a Riemannian metric $\lambda$ on $\mathscr{S}$, and assume $\lambda$ is $\boldsymbol{Q}$-valued. Let $f$ and $g$ be oriented $q$-cochains on $\mathscr{S}$ with values in $\boldsymbol{Q}$. We set

$$
f, g)=\sum_{\sigma} f(\hat{\sigma}) g(\hat{\sigma}) \lambda(\sigma)
$$

where the sum is over all geometric $q$-simplices $\sigma$ of $\mathscr{S}$. We let $C^{q}$ denote the oriented $q$-cochains of $\mathscr{S}$ with values in $\boldsymbol{Q}$. Then (, ) defines a positivedefinite inner product on $C^{q}$. Let $d: C^{q} \rightarrow C^{q+1}$ denote the simplicial coboundary. We let $\delta: C^{q} \rightarrow C^{q-1}$ denote the adjoint of $d$, with respect to (, ) and we set $\Delta=d \delta+\delta d$. Since (,) is positive-definite, it is easy to see that, for $f \in C^{q}$,

$$
\begin{aligned}
d \delta f & =0 \text { implies } \delta f=0, \\
\delta d f & =0 \text { implies } d f=0, \\
\Delta f & =0 \text { implies } d f=0 \text { and } \delta f=0 .
\end{aligned}
$$

We let $\boldsymbol{H}^{q} \subset C^{q}$ be the kernel of $\Delta$ (and we call an element of $\boldsymbol{H}^{q}$ a harmonic cochain). From (2.2) it is elementary to prove (see Kostant [3]):

Proposition (Hodge DeCOMposition). $C^{q}$ has (relative to $\left.(),\right)$ an orthogonal direct sum decomposition

$$
C^{q}=\boldsymbol{H}^{q} \oplus d C^{q-1} \oplus \delta C^{q+1} .
$$


It follows that $\boldsymbol{H}^{q} \cong H^{q}(\mathscr{S}, \boldsymbol{Q})$. Thus to prove $H^{q}(\mathscr{S}, \boldsymbol{Q})=0$, it suffices to prove a vanishing theorem for $\boldsymbol{H}^{q}$. Now for real locally symmetric spaces, Matsushima has reduced the study of harmonic forms to the computation of the minimum eigenvalues of certain curvature transformations (see [4]). By analogy, we might hope that we can reduce the study of $\boldsymbol{H}^{q}$ to the computation of the minimum eigenvalue of certain combinatorial curvature transformations. Indeed, for $\mathscr{S}=\Gamma \backslash \mathscr{T}$, we can do exactly that for suitably defined curvature transformations. We can then estimate the minimum eigenvalue well enough to prove Theorem 1.

Presently we shall define our curvature transformation for $q<l$ (for an arbitrary finite simplicial complex with Riemannian metric). First we make some preliminary definitions. By the unit sphere $\Sigma$ about a vertex $\sigma$ in $\mathscr{S}$, we will mean the boundary of the star of $\sigma$. Now for $q>0$, every $q$-simplex $\tau$ of $\mathscr{S}$, having $\sigma$ as a vertex, determines a $(q-1)$-simplex $\tau^{\prime}$ of $\Sigma$. We set $\lambda^{\prime}\left(\tau^{\prime}\right)=\lambda(\tau)$; then $\lambda^{\prime}$ is a Riemannian metric on $\Sigma$. We let $d^{\prime}$ denote the simplicial coboundary on $\Sigma$ and $\delta^{\prime}$ the adjoint of $d^{\prime}$ (with respect to $\lambda^{\prime}$ ). Then for $q<l$ our curvature transformation (at $\sigma$ ) is just $\delta^{\prime} d^{\prime}$ acting on the space of $\boldsymbol{Q}$-valued $q$-cochains of $\Sigma$ (orthogonal to the constant cochains if $q=1$ ).

For example, if $G_{k_{v}}=S L_{3}\left(k_{v}\right)$, and $F$ denotes the residue class field of $k_{v}$, then every unit sphere $\Sigma$ in $\Gamma \backslash \mathscr{T}$ is isomorphic as a simplicial complex to $\Sigma_{0}$, which we now define. Let $F^{3}$ denote the three-dimensional vector space over $F$. The set of vertices of $\Sigma_{0}$ will be the set of all lines and planes (containing the origin) in $F^{3}$. Two vertices span a one-simplex ( $\Sigma_{0}$ is onedimensional) if and only if one is a line, the other a plane, and the line is contained in the plane. We may take $\lambda^{\prime}$ to be identically one. In this case $O$. Rothaus showed me the eigenvalues of $\delta^{\prime} d^{\prime}$. They are $0,2,1$ $\pm q^{1 / 2} /(q+1)$, where $q$ is the cardinality of $F$. For general rank 2 groups $G$, the needed computation is contained in Feit-Higman [2]. One can also use the generators and relations of the Hecke algebra. Finally, the general case is handled by an induction argument, starting with the Feit-Higman result.

\section{REFERENCES}

1. F. Bruhat and J. Tits, Groupes algébriques simple sur un corps local, Proc. Conf. Local Fields (Driebergen, 1966), Springer, Berlin, 1967, pp. 23-36. MR 37 \#6396.

2. W. Feit and G. Higman, The nonexistence of certain generalized polygons, J. Algebra 1 (1964), 114-131. MR 30 \#1189.

3. B. Kostant, Lie algebra cohomology and the generalized Borel-Weil theorem, Ann. of Math. (2) 74 (1961), 329-387. MR 26 \#265.

4. Y. Matsushima, On Betti numbers of compact, locally symmetric Riemannian manifolds, Osaka Math. J. 14 (1962), 1-20. MR 25 \#4549.

5. W. Schmid, On a conjecture of Langlands, Ann. of Math. (2) 93 (1971), 1-42.

6. J. A. Shalika, On the space of cusp forms of a p-adic Chevalley group, Ann. of Math. (2) 92 (1970), 262-278.

Department of Mathematics, Cornell University, Ithaca, New York 14850

Current address: Department of Mathematics, Columbia University, Columbia, NEW YORK, 10027. 